\title{
Against the view that consciousness and attention are fully dissociable
}

\section{Giorgio Marchetti *}

Pozzo d'Adda, Milan, Italy

Edited by:

Naotsugu Tsuchiya, RIKEN, Japan

Reviewed by:

David Rosenthal, City University of New York, USA

Ramakrishna Chakravarthi, Université

Paul Sabatier, France

*Correspondence:

Giorgio Marchetti, www.mind-

consciousness-language.com, via

Guido Rossa, 8, Pozzo d'Adda, Milan,

Italy.

e-mail: giorgio.marchetti@nsn.com
In this paper, I will try to show that the idea that there can be consciousness without some form of attention, and high-level top-down attention without consciousness, originates from a failure to notice the varieties of forms that top-down attention and consciousness can assume. I will present evidence that: there are various forms of attention and consciousness; not all forms of attention produce the same kind of consciousness; not all forms of consciousness are produced by the same kind of attention; there can be low-level attention (or preliminary attention), whether of an endogenous or exogenous kind, without consciousness; attention cannot be considered the same thing as consciousness.

\section{Keywords: attention, consciousness, visual perception}

\section{INTRODUCTION}

In the controversial and unresolved debate about the relationship between attention and consciousness, Koch and Tsuchiya's (2006) article certainly had the merit of posing the problem of the relationship between attention and consciousness in a very clear, schematic, and provocative way. They maintained that top-down attention and consciousness are distinct phenomena that need not occur together, and presented evidence that all the following four cases are possible:

(1) top-down attention with consciousness

(2) top-down attention without consciousness

(3) consciousness without top-down attention

(4) no top-down attention - no consciousness

Albeit focused on a specific kind of attention, that is, top-down attention, this fourfold classification offers an ideal comprehensive framework that can be generalized in order to study the possible relationships between all forms of consciousness and attention.

I will try to show here that, even if attention cannot be considered the same thing as consciousness, some form of attention is always necessary for consciousness, and that high-level topdown attention always implies some form of consciousness (or alternatively, that there can be low-level attention or preliminary attention, whether of an exogenous or endogenous kind, without consciousness). More in general, I will also present evidence that: there are various forms of attention and consciousness; not all forms of attention produce the same kind of consciousness; not all forms of consciousness are produced by the same kind of attention.

\section{POSITIVE EVIDENCE OF THE CLOSE CORRELATION BETWEEN ATTENTION AND CONSCIOUSNESS}

The idea that attention is strictly linked to consciousness is not new (James, 1890; Posner, 1994; O’Regan and Noë, 2001). Indeed, the idea is quite intuitive, if we consider what is thought to be one of the main characteristics of attention: its selective power. When we attend to a certain object or part of an object, we are able to isolate it from the other objects or parts, so that our conscious mind is completely and exclusively possessed and "filled" by it (La Berge, 1995). Even though this does not prove that attention is necessary or sufficient for consciousness, it shows that there is a direct connection between attention and consciousness: how we pay attention to the world is highly correlated with how the world appears to us. Moreover, well-known psychological phenomena demonstrate that attention modulates perception, directly influencing the way we consciously experience the world. Let's consider some evidence from psychological studies. These examples of a tight correlation between attention and consciousness should be taken into account for a possible falsification by all those who maintain that attention and consciousness are fully dissociable.

\section{PSYCHOLOGICAL STUDIES OF VISUAL PERCEPTION}

Carrasco's (2011) work provides empirical evidence that attention alters phenomenal appearance: it boosts the apparent stimulus contrast. For example, Liu et al.'s (2009) study shows that voluntary (endogenous, sustained) covert spatial attention alters the appearance of objects. In this study, which provides a phenomenological correlate of the effect of voluntary attention on perception, voluntary attention increases the perceived contrast of suprathreshold stimuli: attending to a peripheral location makes a cued 29\%-contrast stimulus and an uncued 36\%-contrast stimulus both subjectively equivalent to a $32 \%$-contrast stimulus.

Likewise, Carrasco et al. (2004) show that when the transient covert attention (which is the stimulus-driven, exogenous, involuntary capture of attention by an abrupt, salient peripheral cue) of observers is drawn to a suprathreshold stimulus via a peripheral cue, they report this stimulus as being higher in contrast than it really is, thus indicating a change in appearance with attention (however, it should be noted that this work was not free of controversies: see Schneider, 2006; Prinzmetal et al., 2008; Schneider and Komlos, 2008. For the answer, see Carrasco et al., 2008; Carrasco, 2011). 


\section{PSYCHOLOGICAL STUDIES OF THE PERCEPTION OF TIME}

Important evidence that attention modulates perception also comes from the domain of the studies of time perception. The phenomenon of prior-entry, for example, shows that when a person attends to a stimulus, he or she perceives it as having occurred earlier in time than if he or she was not attending to it (Shore et al., 2001). Experiments on duration judgments in which subjects are asked to prospectively judge the duration of the time period they have to perform a certain task, reveal that judged time decreases linearly with the increased processing demands of non-durational information, and that experienced duration increases to the extent that subjects can allocate more attentional resources to the flow of time itself (Hicks et al., 1976, 1977; Brown, 1985; Coull et al., 2004). In short, a higher amount of attention allocated to the passage of time itself produces a lengthening of the experienced duration. A similar attentional effect results when attention is directed in advance to one of two possible stimulus sources: Mattes and Ulrich (1998) show that subjects judge a stimulus as being longer when it appears at the precued stimulus source than when it appears at the uncued one; that is, directed attention prolongs the perceived duration of a stimulus.

\section{INATTENTIONAL BLINDNESS (IB) AND CHANGE-BLINDNESS}

The idea that attention is necessary for consciousness has received strong support from the work of Mack and Rock (1998) and Rensink et al. (1997). In Mack and Rock's (1998) experiments, the subject's attention was engaged in a task (such as identifying the longer arm of a cross briefly presented on the screen and centered at about $2^{\circ}$ from fixation). After some trials, an unexpected, unsearched critical stimulus (for example, a black circle) was presented at fixation, and subjects were asked whether they had seen anything that had not been on the screen in the earlier trials. Between 60 and $80 \%$ of the subjects failed to detect the critical stimulus. A comparison between reports of the critical stimulus in the inattention, critical trials (where subjects were told to pay attention to the cross, but were not told that a critical stimulus would appear) and those in full attention control trials (where subjects were told to ignore the cross, and to report only what else they saw on the screen when the cross was present), confirmed that focal attention is clearly implicated in conscious perception. More in general, Mack and Rock's experiments show that subjects tend to be blind to a critical stimulus that appears either at, or close to, fixation when they are not searching for it, when they are occupied with a task that engages their attention, and when it is located outside the boundaries of the area on which attention is directed. These findings do not imply that there is no implicit, unconscious perception, but only that there is no explicit, conscious perception prior to the engagement of focal attention. Stimuli to which subjects are inattentionally blind, can be implicitly, unconsciously perceived. In order to bring them into consciousness, they must be attentionally processed.

Inattentional blindness (IB) results, however, could be subject to alternative interpretations involving processes other than attention, such as expectation and memory (Lavie, 2006a). Firstly, the critical stimulus is expected in control trials, and subjects are likely to look for it intentionally: therefore, the comparison of control and critical trials may confound effects of attention with effects of expectations. Secondly, while in control trials awareness reports are made immediately following display presentation, in critical trials they are made after a task response and a surprise awareness question in critical trials: therefore, reduced rates of awareness in critical vs. control trials may reflect higher rates of forgetting during the longer delay from display presentation until the awareness question in the critical trials ${ }^{1}$.

The alternative explanation based on expectation was ruled out by Cartwright-Finch and Lavie's (2007) study, which, manipulating perceptual load within Mack and Rock's (1998) IB paradigm, compared the rates of IB not so much between the critical and control trials, as between critical trials of different levels of load (that is, the critical stimulus was equally task-irrelevant and equally unexpected across the varying levels of perceptual load). Cartwright-Finch and Lavie found that the level of perceptual load in the task determined the rates of IB: whereas subjects were often aware of the irrelevant stimuli in situations of low load, they failed to notice the irrelevant stimulus in situations of high load. This result shows that the level of attention available for the processing of the task-irrelevant stimulus determines whether the stimulus can be consciously perceived.

The alternative explanation based on memory is ruled out by Rensink et al.'s (1997) study, which, unlike the IB paradigm, does not rely on a retrospective question about an unexpected stimulus: indeed, subjects are instructed in advance that their task is to detect whether a change occurred between two successive images and report about it immediately after seeing the images. Rensink et al. (1997) developed the "change detection flicker task" paradigm [which is a variant of the change-blindness (CB) paradigm: see for example Simons and Levin, 1997, 1998]: an original image $A$ repeatedly alternates with a modified image $A^{\prime}$, with brief blank fields placed between successive images; differences between original and modified images are highly visible; subjects freely view the flickering display and hit a key when they perceive the change. In order to prevent guessing, subjects are asked to correctly report the type of change and describe the changing part of the scene. Because the stimuli are available for long stretches of time and no eye movements are required, the flicker paradigm provides the best opportunity possible for an observer to build a representation conducive to perceiving changes in a scene. The CB found with the brief-display techniques adopted by previous experimental paradigms might have been caused by insufficient time to build an adequate representation of the scene; saccade-contingent $\mathrm{CB}$ might have been caused by disruptions due to eye movements.

\footnotetext{
${ }^{1} \mathrm{IB}$ results can also be subject to alternative interpretations involving different forms of consciousness. As we will see, it is possible to distinguish between a "primary" form of consciousness and a "higher-order, reflective" form of consciousness (Bartolomeo, 2008). It can be argued that in IB the subjects are aware of the critical stimulus (that is, they have a primary consciousness of the critical stimulus), but are not able to verbalize it (that is, they lack a reflective consciousness necessary to verbally describe the critical stimulus). However, it should be noted that Mack and Rock, 1998, pp. 197-204) performed some experiments explicitly aimed at verifying the hypothesis that the subject "has fleetingly perceived the critical stimulus, but fails to encode it." The experiments showed that the subjects were not even faintly aware of the critical stimulus, and that many subjects did not even see a critical stimulus visible for a total period of $700 \mathrm{~ms}$ (Mack and Rock, 1998, pp. 202-204), which contradicts the hypothesis that the subjects were "aware" of the critical stimulus, but were unable to verbalize it.
} 
Both of these factors are eliminated in the flicker paradigm, so that if they are indeed the cause of the difficulties, perception of change should now become easy. But if attention is the key factor, a different outcome would be expected. And it is precisely this different outcome that Rensink et al.'s study reveals. Their experiments show that identification of changes becomes extremely difficult, even when changes are large and made repeatedly: under flicker conditions subjects take a surprisingly long time to perceive large changes in images of real-world scenes. This difficulty is due neither to a disruption of the information received nor to a disruption of its storage: it does however depend greatly on attention. The role of attention is further confirmed by the findings that: cueing the changing object removes any difficulty in detecting change (Rensink et al., 1997); objects that capture attention, either by virtue of containing a singleton feature, or by virtue of their significant socio-biological meaning, do not suffer from CB (Ro et al., 2001); the rate of change detection critically depends on the level of load in the search task, that is, subjects fail to detect the change far more often in the condition of high vs. low load in the search task (Lavie, 2006a,b).

Rensink et al.'s (1997) experiments also answer most of the criticisms raised by Mole (2008). According to Mole, what Mack and Rock's (1998) work shows is only that there are some circumstances in which attention is necessary for consciousness: that is, cases in which the stimulus is presented unexpectedly, for a fifth of a second, concurrently with something else that one is attending to, in an unfamiliar experimental paradigm, and followed by a pattern mask. As Rensink et al.'s (1997) experiments show, attention is also necessary with familiar, and available for long stretches of time stimuli, when the subject is aware of the kind of stimulus, and even when the subject is aware of the types of change possible and is given practice trials before the experiment.

Mole's (2008) criticisms, however, seem to imply a more general kind of criticism based on "some relatively uncontroversial features of the epistemology of perception." According to these considerations from the epistemology of perception, Mack and Rock's (1998) and Rensink et al.'s (1997) experiments would not appear to show that attention is always necessary for consciousness, but only that attention is necessary when one's experience is to provide one with knowledge of the sort probed by the experimenter's questions in a $\mathrm{CB}$ or IB experiment. In other words, $\mathrm{CB}$ and IB experiments suffer from the defect of not giving the possibility of independently ascertaining whether attention is always necessary for consciousness: as they are designed, these experiments would only reveal that attention is necessary for consciously detecting changes or unexpected stimuli but not for other cases. According to this view, a subject who has not attended to the changing item in the CB pictures or to the unexpected stimuli in the IB experiment, could theoretically have some kind of conscious experience, but the experience does not have the "structured content" needed to provide the subject with knowledge of the fact that the thing is changing or unexpectedly appearing.

I think that Mole's (2008) epistemological considerations are legitimate and that they deserve to be properly addressed. In order to do so, I will analyze some experiments that either have been explicitly conceived and designed to prove that there can be consciousness in the absence of attention or have been interpreted as evidence that attention is not necessary for consciousness. The analysis will show that even such experiments substantially fail to prove the thesis that attention is not necessary for consciousness, and consequently that an answer can also be provided to Mole's epistemological considerations (at least until contrary evidence is offered).

\section{EXPERIMENTS AIMED AT DEMONSTRATING CONSCIOUSNESS IN THE ABSENCE OF ATTENTION}

The idea that attention is necessary for consciousness is not universally accepted (Umiltà, 1994; Baars, 1997; Hardcastle, 1997; Lamme, 2003; Koch and Tsuchiya, 2006). A very strong and paradigmatic version of the view that attention and consciousness are distinct phenomena is that held by Koch and Tsuchiya (2006). They maintain that "top-down attention and consciousness are distinct phenomena that need not occur together and that can be manipulated using distinct paradigms" (Koch and Tsuchiya, 2006, p. 16).

Given the strong position held by Koch and Tsuchiya (2006), I will start my analysis of the evidence that there can be consciousness in the absence of attention by considering the examples and arguments they put forward in favor of their hypothesis. To do so, I will proceed by separately considering some of the perceptual phenomena and behaviors they have presented as evidence of the dissociation between attention and consciousness. Additionally, I will also consider some complementary examples and arguments proposed by other researchers in support of the view that attention is not necessary for consciousness.

As my analysis will show, Koch and Tsuchiya's (2006) view that consciousness can be dissociated from top-down attention is only partly true, and needs further specification. There are cases of consciousness in the absence of a certain form of top-down attention, but in the presence of some other form of top-down attention. There are cases of consciousness in the absence of top-down attention but in the presence of some other form of attention, such as bottom-up attention. But there are never cases of consciousness in complete absence of some form of attention.

In my view, Koch and Tsuchiya's (2006) position that consciousness can be dissociated from top-down attention mainly originates from a failure to notice the varieties of forms that specifically topdown attention and more in general attention and consciousness can assume. As many authors have highlighted (Bartolomeo, 2008; Posner, 2008; Srinivasan, 2008; Koivisto et al., 2009; De Brigard and Prinz, 2010; Kouider et al., 2010), in order to correctly understand the relationship between attention and consciousness, it is essential to duly take into account the varieties and complexity of forms of attention and consciousness: overlooking this factor may lead to the wrong view that there can be consciousness without attention.

Indeed, top-down attention can assume at least two different forms: focused attention and diffused or distributed attention (Treisman, 2006; Demeyere and Humphreys, 2007; Srinivasan et al., 2009; Alvarez, 2011); it can, up to a certain extent, be split between different perceptual and processing modalities (Pashler, 1998); it can be both widely distributed for relatively long time periods in a certain location (preparatory attention) and narrowly distributed in another location for shorter periods (selective attention; La Berge, 1995); it varies according to the perceptual 
Table 1 | A categorization of top-down and bottom-up attention.

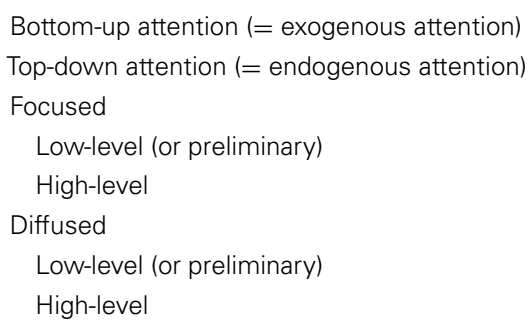

load (Lavie, 1995); the total amount of allocated attention can vary from a minimum, "preliminary" level (Velmans, 1991) up to a maximum limit defined by the available attentional resources, according to motivation and arousal (Kahneman, 1973); and so on. Moreover, forms of attention exist (such as bottom-up) that differ from top-down attention and which also produce consciousness.

In Table 1, I propose a possible finer categorization of top-down attention based on the amount of allocated attention (low-level/high-level) and the spatial extent of attention (focused/diffused). It is important to note that the distinction between focused and diffused attention does not imply two separate, opposite dimensions but rather, as Srinivasan (2008) suggests, two ends of a continuum in which the focus varies. The use of focused vs. diffused attention depends on the specific task and motivation (such as tracking a single object vs. multiple objects). Likewise, the distinction between high- and low-level attention represents two extremes of a continuum in which the amount of allocated attentional resources varies. Moreover, as showed by Kahneman (1973), the total amount of attentional resources that is allocated, is not constant for every task, but can vary according to various factors, such as the difficulty and novelty of the task, the arousal level, the expectations, etc. Usually, high-level topdown attention is used with new, interesting, or complex tasks. On the contrary, low-level top-down attention may occur with uninteresting, repetitive, or daily tasks, the secondary task in a dual-task paradigm when both tasks draw upon the same pool of resources, and when the stimulus duration does not allow for top-down attention to be fully deployed.

Similarly, a general awareness of our environment (ambient awareness) can be distinguished from a more detailed focal awareness of a scene (focal awareness; Iwasaki, 1993); a form of primary consciousness, including an awareness of the world and mental images, but not a concept of self, can be distinguished from a form of higher-order consciousness, including self-awareness, a sense of time, and language (Edelman, 1989); forms of spatial awareness can be distinguished from more reflective forms of consciousness based on intellectual acknowledgment (Bartolomeo, 2008); consciousness of sensory qualities differs from volition, which in turn differs from the simple conscious state, which neurology associates with the concept of arousal and the diurnal cycle of sleep and wake (Posner, 2008); an evolutionary primitive form of consciousness, relatively independent of voluntary attention (anoetic consciousness), can be distinguished from a more recent and complex form of self-awareness that requires the use voluntary attention (autonoetic consciousness; Vandekerckhove and Panksepp, 2009);

\section{Table 2 | A classification of consciousness}

(a) Anoetic consciousness (pre-reflective affective and sensorial perceptual consciousness)

(b) Noetic consciousness (semantic memory, but not yet access to a full awareness of one's own ongoing subjective experience)

(c) Autonoetic consciousness (explicit self-awareness)

conscious phenomena can be differently classified according to the time scales according to which events are integrated (for example, Wittmann, 2011, identifies three levels: functional moment, experienced moment, and mental presence).

In Table 2, I propose a possible classification of consciousness based on Tulving (1985) and Vandekerckhove and Panksepp (2009).

\section{ATTENTIONAL BLINK}

Koch and Tsuchiya (2006) quote Olivers and Nieuwenhuis' (2005) study on the attentional blink. The attentional blink (Shapiro et al., 1994) occurs when subjects view rapid serial visual presentations of a series of stimuli presented in the same location, usually at rates of approximately $100 \mathrm{~ms}$ per item. Subjects have to detect two target stimuli, T1 and T2; T1 appears first and is followed by T2, which may appear immediately after $\mathrm{T} 1$ or at some other point in the sequence after $\mathrm{T} 1$, with distractors presented between $\mathrm{T} 1$ and $\mathrm{T} 2$ (that is, the temporal lag between T1 and T2 can vary). The blink effect refers to a decrement in detection of T2: the basic finding is that the decrement is often greatest when T2 occurs not immediately after $\mathrm{T} 1$ (position $n+1$ ), but rather somewhere around positions $n+2$ through $n+5$ (that is, when there are one or more distractors between $\mathrm{T} 1$ and $\mathrm{T} 2$ ). The performance improves with a higher lag and reaches asymptote around $n+6$ or $n+7$. A possible explanation of the attentional blink is that processing of $\mathrm{T} 1$ takes up limited attentional resources: as a result, either access to these resources is denied for T2 or the representation of T2 is so vulnerable that it easily suffers from the interference of temporally surrounding distractors (for a review of the alternative theoretical accounts of the AB, see Shapiro et al., 1997; Di Lollo et al., 2005).

Olivers and Nieuwenhuis' (2005) study, which was motivated by the observation that participants in previous experiments reported rather counterintuitively improved T2 performance when being somewhat unfocused on the task, shows that the attentional blink is significantly ameliorated when observers are concurrently engaged in distracting mental activity, such as free-associating on a task-irrelevant theme or listening to music. The experiment suggests that under conditions of rapid visual presentation, target detection may benefit from a diffusion of attention.

Koch and Tsuchiya (2006) have interpreted Olivers and Nieuwenhuis' (2005) findings as indicating that top-down attention and consciousness can oppose each other. However, as Srinivasan (2008) argues, there is an alternative interpretation based on the concept of differential attentional strategy, which is more economical than Koch and Tsuchiya's because it does not require two different processes (one for attention and another for consciousness) to explain the same phenomenon, but only one process (attention). The alternative interpretation is that under certain 
conditions, such as when subjects know that they need to consider a large number of items in order to report the second target stimulus in an experiment on attentional blink, diffused attention may turn out to be a better strategy than focused attention. This also corresponds to one of the three explanations that Olivers and Nieuwenhuis put forward to account for the phenomenon they found: it may have actually been the additional task itself that induced a more distributed state of attention. As attention widened to incorporate the extra task, it may have also widened temporally and thus included $\mathrm{T} 2$ in the series of stimuli.

However, this alternative interpretation does not account for the overall improvement in T1 performance in the music condition. In fact, diffused attention alone cannot explain how T1 detection performance can also improve as a result of an additional task. A more reliable interpretation seems to rely not only on diffused attention, but also on a temporary increase of the allocated attentional resources due to the difficulty of the task. This temporary increase seems to be implied by the two other possible explanations offered Olivers and Nieuwenhuis (2005). According to the first, the effects may be related to arousal: decreased or increased arousal - as may have occurred in the free-association and music conditions - may have made the attentional system more susceptible to other input, including T2. According to the second, thinking about one's holiday or listening to music may have induced a positive affective state, which has shown to improve performance on several cognitive tasks.

\section{ANIMAL AND GENDER DETECTION IN DUAL-TASK}

Koch and Tsuchiya (2006) also quote Li et al.'s (2002) work, which shows that subjects can rapidly detect animals or vehicles in briefly presented novel natural scenes while simultaneously performing another attentionally demanding task, and Reddy et al.'s (2004) work, which, comparing how subjects perform on a face-gender discrimination task carried out in the single task condition with the same task carried out in the dual-task condition with a known attentional demanding task (five-letter T/L discrimination), shows that the face-gender discrimination task can be performed equally well under the two conditions.

According to Koch and Tsuchiya, 2006, p.19), this kind of evidence shows that "although it cannot be said with certainty that observers do not deploy some top-down attention to the peripheral target in dual-task experiments that require training and concentration (i.e., high arousal), it seems that subjects can perform certain discriminations in the near absence of top-down attention $^{2}$."

\footnotetext{
${ }^{2}$ Indeed, it is the very possibility that subjects can deploy some form of attention to the peripheral stimulus in dual-task experiments, that leads van Boxtel et al., 2010a, p. 6) to admit that "the major obstacle for solving the question of whether there is consciousness without attention is that there is no objective psychophysical way to unambiguously determine a state of 'complete absence of attention.' It is not known whether the conditions measured in the dual-task paradigms are cases of 'very low attention' or 'no attention' "See also the observations made by Kouider et al., 2010, p. 304): not only "it seems to be extremely difficult, if not impossible, to assess whether subjects consciously perceive objects in the periphery without relying on some form of access," but also "the possibility of consciousness without attention is usually based on a restrictive definition that does not take into account the possibility of residual attention at lower (i.e. sensory, non-conceptual) levels of processing."
}

The view that this kind of evidence shows the independence of consciousness from attention, has been challenged on various grounds, even though not all the argumentations seem equally convincing.

As observed by Taylor and Fragopanagos (2007), in these experiments the subjects underwent up to $10 \mathrm{~h}$ of prior training on the stimuli, which makes it highly plausible that the subjects learnt to develop an automatic route to respond to the peripheral stimuli to which they were exposed. However, it should be noted that in their Experiment 4, Reddy et al. (2004) explicitly controlled whether there was a confounding effect of training in the face-gender discrimination task (they tested subjects who had been trained on a completely different dual-task experiment, that is, natural scene categorization: animal vs. non-animal or vehicle vs. nonvehicle), without finding any significant difference in performance compared with the previous experiments.

Taylor and Fragopanagos (2007) also put forward the hypothesis that the subjects were able to use multiple foci of attention to detect the presence of both the peripheral target as well as the main central one (McMains and Somers, 2004). While not completely improbable, the hypothesis seems however questionable because it does not explain why the subjects did not improve their performance in the peripheral task by taking advantage of the free extra-resources made available when asked to ignore the central task (when the subjects performed the face-gender discrimination task alone, their performance was not significantly different from performance on this task in the dual-task condition).

I think that to correctly interpret experiments such as Reddy et al. (2004) one should consider the possibilities of temporary increasing the amount of allocated attentional resources, and varying the spatial extension of the focus of attention. Just as for the case of the attentional blink, it is likely that the highly demanding task implied by dual-task paradigm induces subjects to adopt diffused attention as a better strategy than focused attention, and to increase the total amount of allocated attentional resources (compared to the single task). Moreover, it is known that meaningful stimuli such as faces capture exogenous attention. Therefore, Reddy et al.'s (2004) experiments can be interpreted as a combination of exogenous attention and increased, diffused top-down attention.

\section{GIST}

Koch and Tsuchiya (2006) observe that we are aware of the gist of a scene or of our surrounding environment even when we are not paying attention to it: "In a mere $30 \mathrm{~ms}$ presentation time, the gist of a scene can be apprehended. This is insufficient time for top-down attention to play much of a role. Furthermore, because gist is a property associated with the entire image, any process that locally enhances features, such as focal attention, will be of limited use" (p. 18).

Here, Koch and Tsuchiya (2006) seem to conflate top-down attention with focal attention. As we have seen, top-down attention can have two forms: focused or diffused. Even if focal attention is absent or nearly absent, one cannot exclude that some form of diffused attention may be involved, which allows us to capture the gist of a scene. This is also De Brigard and Prinz's (2010) view, who observe that there is no reason to think that attention is 
absent, but rather that it is more plausible to think that attention is only diminished. Indeed, the view that attention is necessary for consciousness predicts such findings very well: when attention is nearly absent, we are aware of far less than when it is fully deployed. This is why the gist is perceived and no more.

Alternatively, it is also possible to conceive the phenomenon of gist reported by Koch and Tsuchiya (2006) as evidence of the existence of a specific form of consciousness: which Bartolomeo (2008) calls "primary consciousness." Primary consciousness refers to the basic condition of being aware of something: as such, it must be distinguished from a higher-order, reflective form of consciousness, which can involve linguistic abilities and allows subjects to perceive and describe their own actions and thoughts. Not always what enters primary consciousness also enters the higher-order form of reflective consciousness: overlooking this fact may sometimes lead to the wrong observation that the absence of a verbal report means the absence of consciousness tout court. Bartolomeo (2008, p. 17) illustrates the difference between the two forms of consciousness by quoting an example given by MerleauPonty "of someone who enters a room and feels an impression of disorder, only to later discover that this impression came from a crooked picture on the wall. Before discovering that, this person's consciousness was 'living things that it could not spell out.' This would by no means imply that the first impression on entering the room was unconscious. Rather, the crooked picture generated a form of consciousness whose source was not immediately amenable to verbal description."

The existence of this form of primary consciousness is supported by findings such as Landman et al.'s (2003), who show that people's memory of a visual image has a large capacity representation (more than four items) and remains intact for at least 1200-1500 ms after the stimulus has disappeared.

\section{POP-OUT AND THE COCKTAIL PARTY EFFECT}

Treisman and Gelade (1980) showed that when subjects search for a target defined by a conjunction of properties (say a red T) amongst a number of non-target items or distractors (say, red Xs and green Ts), search time increases linearly with the number of distractors. On the contrary, when subjects search for a target defined by a unique feature (say a red letter among green letters), search time is independent of the number of distractors. In this case, the unique feature is said to "pop-out." The pop-out effect is sometimes interpreted as evidence of the fact that the unique feature "automatically" captures attention, in the sense that subjects can become aware of the target prior to and independently of the focusing their attention to it.

In my view, this interpretation is wrong because in visual search experiments subjects are actively looking for the target (Mack and Rock, 1998). As Most et al., 2005, p. 219) observe: "Although popout search has sometimes been interpreted as evidence that a target has automatically captured attention (...), in a strict sense this kind of evidence is insufficient to infer automatic attention capture. Because the observer is actively looking for the target, his or her attention is presumably broadly and purposefully distributed throughout the display." A first empirical confirmation of the role of top-down attention in pop-out was obtained by Hsieh et al. (2011), who found that top-down attention is necessary for the subliminal pop-out effect to occur (Experiment 2: unseen feature singletons do not recruit attention when subjects are distracted by a rapid sequential visual presentation task while viewing the subliminal pop-out display). Therefore, it seems wrong to consider the pop-out effect as evidence of consciousness in the absence of top-down attention.

A related but slightly different phenomenon - the cocktail party effect - shows more in general that some kind of attention - whether top-down or bottom-up - is always necessary for consciousness. Using a dichotic listening task, where subjects who were presented with two simultaneous messages to both ears via headphones were asked to attend to the message presented to one ear and to ignore the other message presented to the other ear, Moray (1959) found that subjects still recognized their name when it was presented in the unattended ear (the cocktail party effect). Cases such as this, where the subject's name or some other meaningful stimuli can exogenously capture a subject's attention even though the subject does not expect them or have any intention toward them, do not involve top-down attention (subjects are asked to focus on one message and ignore the other message) but rather a form of peripheral, exogenous attention. Indeed, it does not seem so implausible to think that: (a) evolution has endowed us with some mechanism that allows us to quickly orient to salient features of our environment; (b) this mechanism is (at least partly) based on the working of some form of peripheral, exogenous attention, which, being constantly applied and distributed, albeit at low levels of intensity, can be captured when salient stimuli occur.

Umiltà (1994) interprets the cocktail party effect and similar effects as evidence that attention does not coincide with consciousness and that they must be considered as independent systems. In these cases, he argues, the object is perceived consciously in a direct manner, without the intervention of attention.

Umiltà's (1994) argument contrasts with what Mack and Rock (1998) have found. They show that by decreasing the probability that attention is paid to an object, the probability of perceiving its presence is reduced. This also applies to cases of captured or exogenous attention: when the difficulty of capture is increased by reducing the attentional zone or increasing the inhibition of attention, the probability that one's own name is reliably perceived decreases (even if it continues to be seen significantly more often than other stimuli). Moreover, as McCormick (1997) has shown, exogenous cues presented below a subjective threshold of awareness capture attention without awareness.

These facts (as well as other instances of consciousness produced by the sole exogenous attention: Chica et al., 2011) indicate that some kind of attention is always involved in conscious perception. Even objects such as one's own name cannot be perceived without the intervention of some form of attention: they must capture attention to become conscious. There cannot be conscious perception without attention.

\section{ICONIC MEMORY}

Lamme (2003) also proposes that there can be consciousness without attention. In his view, the attentive selection process operates at a later stage than consciousness: attention does not determine whether stimuli reach a conscious state, but determines whether a conscious report about stimuli is possible. In other words, we are 
conscious of many inputs, but without attention this conscious experience cannot be reported: when we view a visual scene, we experience a richness of content that goes beyond what we can report. His model - which presupposes the existence of a short-lived, vulnerable, and not easily reportable form of visual experience, which contrasts with a more stable, reportable form of awareness - parallels: (a) Block's (1996) proposal of the existence of two distinct kinds of awareness: phenomenal and access awareness; and (b) the distinction made in the domain of sensory memory between "iconic memory" and "working memory."

In support of his view, he quotes Becker et al.'s (2000) and Landman et al.'s (2003) change detection experiments. It is known from $\mathrm{CB}$ experiments that the ability of subjects to detect a change in a visually presented array of items is greatly reduced if a blank interstimulus interval (ISI) is inserted between the original array (stimulus 1) and a subsequent array displaying the same items as stimulus 1 except for one item that has changed (stimulus 2 ). It is also known that change detection improves if the item to be changed is cued during the display of stimulus 1 . The new and surprising phenomenon found by Becker et al. (2000) and Landman et al. (2003) is that change detection also improves when the location of the change is cued during the blank ISI. This may lead one to believe that all of the items of stimulus 1 are conscious and remain in consciousness even after the stimulus is removed, until it is overwritten by stimulus 2 .

According to Lamme (2003), attention is a selection process that determines not so much whether stimuli reach consciousness, as whether stimuli can go from phenomenal awareness to access awareness. This model is based on the considerations that:

(i) there are different levels of processing that stimuli can reach. More specifically, there are sensory inputs that: (1) reach a conscious state via the process of attentive selection; (2) do not reach a conscious state when not attended; (3) do not reach consciousness, not even when attended;

(ii) these different levels of processing can be more parsimoniously explained by a model that is based on an early distinction between conscious and unconscious stimuli than by a model that is based on an early distinction between attended and unattended stimuli. Indeed, while the early distinction between attended and unattended stimuli would lead to postulating at least three different processes (one for stimuli that are conscious because attended, one for stimuli that are unconscious because unattended and one for stimuli that are purely unconscious), the early distinction between conscious and unconscious stimuli would lead to postulating only two processes (one for stimuli that are conscious and one for stimuli that are unconscious).

Lamme's (2003) model seems to overlook the fact that both attention and consciousness can assume a variety of forms. For example, when he observes that there are "non-attentional selection mechanisms" that can produce unconscious processing of stimuli, Lamme does not seem to consider the fact that some sort of "preliminary attention" (Velmans, 1991) can also exist, and that preliminary-attended stimuli, despite being processed, might not be consciously experienced. By overlooking this fact he mistakes unconscious processing for preliminary-attended processing. Moreover, as shown by Bahrami et al. (2008), attention can also act on stimuli that have not reached awareness: stimulus competition for the allocation of attentional capacity occurs regardless of whether or not the observer is conscious of the stimulus representations.

Therefore, it certainly seems to be more plausible and economical to propose a model based on the notion that attention is necessary for consciousness than a model based on the idea that attention is not necessary for consciousness: while the latter implies two processes (one for stimuli that are conscious and one for stimuli that are unconscious), the former needs only one process (stimuli are attended: various levels and types of attention are possible).

This latter view is supported by work. Koivisto and Revonsuo found that "visual awareness negativity" (VAN, which they consider to be the most reliably and consistently observed ERP correlate for subjective visual awareness of a stimulus, vs. a stimulus that does not enter awareness) and visual awareness are dependent on spatial attention (see also Koivisto et al., 2009). According to Koivisto and Revonsuo's, 2010, p. 932), this suggests that iconic processing is not entirely independent of attention: "the visual icon has spatial boundaries limited by the scope and boundaries of spatial attention. Outside the boundaries of spatial attention, there is no visual phenomenology - the spatial boundaries of the visual icon are the spatial boundaries of momentary visual phenomenal experiences."

The finding that change detection improves when the location of the change is cued during the blank ISI (Becker et al., 2000), in my opinion does not show that there can be consciousness without attention; rather, it only confirms that: (a) there is an early component of attention - namely, the exogenous one (Nakayama and Mackeben, 1989) - that can capture a specific item in iconic memory if sufficient time is afforded (change detection and identification tend to worsen at longer ISIs between the offset of stimulus 1 and the onset of the cue); (b) once attention has captured the item, the item is (or can be) transferred to a short-term-memory buffer, where it may be compared with a later-occurring item, thus leading to change detection (change detection and identification tend to improve at longer ISIs between the offset of the cue and the onset of stimulus 2).

Lamme's (2003) idea that when we view a visual scene we experience a richness of content that goes beyond what we can report was questioned because the $\mathrm{CB}$ experiments show that viewers are over-confident about their capacities and suffer from an "illusion of seeing": when viewing a scene, viewers who claim to perceive the entire visual scene, actually fail to notice important changes of the elements of the scene. As argued by O'Regan and Noë (2001), the "illusion of seeing" might arise because viewers know that they can, at will, orient attention to any location and obtain information from it (for a similar view, see also Dehaene et al., 2006; for an alternative explanation of the illusion of seeing, see Kouider et al., 2010).

Despite this criticism, however, I think that Lamme's (2003) idea of the "richness of content" is not fully incompatible with an alternative and equally plausible interpretation of Becker et al.'s (2000) and Landman et al.'s (2003) findings, based on the view 
that there cannot be consciousness without attention. According to this alternative interpretation, the viewer's initial application of attention to a presented array of items triggers a "primary" (nonverbalized), rich form of consciousness (Bartolomeo, 2008) of the visual scene. Subsequently, the content of the primary consciousness can be verbalized thanks to the deployment, via the cue, of an additional amount of attention.

\section{EXPERIMENTS AIMED AT DEMONSTRATING ATTENTION IN THE ABSENCE OF CONSCIOUSNESS}

The fact that attention is necessary for consciousness does not imply that attention generates or can modulate only conscious phenomena: it can also generate and modulate unconscious ones (which in turn can influence or bias both which and how stimuli will be subsequently consciously perceived: for a review, see Merikle et al., 2001). For example, Naccache et al. (2002) demonstrate that it is possible to elicit unconscious priming in a numbercomparison task, but only if the subject's temporal attention is allocated to the time window during which the prime-target pair is presented: unconscious priming vanishes when temporal attention is focused away from this time window. Likewise Montaser-Kousari and Rajimehr (2005) found that attention subliminally modulates and enhances adaptation to illusory lines in the crowding condition even if the crowded item cannot be selected attentively for further processing and is not consciously perceived. Sumner et al. (2006) showed that attention modulates neural sensorimotor processes that are entirely separate from those supporting conscious perception. Bahrami et al. (2008) manipulated perceptual load for a task presented at fixation and assessed orientation specific adaptation to invisible, peripheral tilted gratings that were irrelevant to the task [peripheral gratings were rendered invisible by inter-ocular, continuous flash suppression (CFS)]. They showed that in tasks of low perceptual load any spare capacity from relevant stimulus processing spills over to the processing of irrelevant stimuli (producing orientation specific adaptation) regardless of whether or not subjects are conscious of the representations.

The fact that attention can also generate unconscious phenomena is not per se evidence of the fact that there can be top-down attention without consciousness. As we will see, the view that there can be top-down attention without consciousness will prove to be incorrect: actually, there can be top-down attention without consciousness only when the former is low-level (that is, it does not reach a certain threshold level). However implausible, this view can originate from either confusing the perception of absence with the absence of perception or overlooking the existence of the various forms of attention and consciousness.

Let's first examine some of the examples that were put forward to support the view that there can be top-down attention without consciousness, and then consider whether there is any kind of attention that can be dissociated from consciousness.

\section{CONFUSING THE PERCEPTION OF ABSENCE WITH THE ABSENCE OF PERCEPTION}

Koch and Tsuchiya (2006) maintain that top-down attention and consciousness are dissociated not only because there can be consciousness in the absence of top-down attention, but also because there can be top-down attention in the absence of consciousness. They state that "Subjects can attend to a location for many seconds and yet fail to see one or more attributes of an object at that location" (Koch and Tsuchiya, 2006, p. 17). Likewise, Mack and Rock, 1998, p. 245) state that "It is not an uncommon experience to be looking for something or keenly awaiting its appearance in the absence of perceiving it (...). Both the looking for and the awaiting are part of what we mean by attention in our ordinary language, but in cases such as these the looking for is not associated with any perception."

Generally speaking, I think that when one states that there can be attention without consciousness or without perception, one should carefully specify what the expressions "without consciousness" or "without perception" imply. Indeed, there are cases in which attention can also generate and modulate unconscious phenomena and perceptual illusions. However, when such cases occur they do not imply that there is no consciousness or perception at all. Rather, they imply that a person can be aware of something without being aware of something else, or even that a person can be aware of not being aware of something. The fact that in some cases one can focus one's attention to something without perceiving it does not imply that one does not perceive anything at all: rather it means that one perceives something else, or that one perceives the absence of the thing one is focusing on. As Mole (2008) correctly observes, cases in which one is on the lookout for something that does not appear, are not cases of attention without perception; rather, these are cases where one is perceiving that nothing has yet occurred. Overlooking this means mistaking the perception of absence for the absence of perception.

Therefore, in my opinion, experiments such as MontaserKousari and Rajimehr's (2005) provide evidence not so much of top-down attention in the absence of consciousness, as of "topdown attention in the absence of consciousness of something, but in presence of consciousness of something else," or of "top-down attention with consciousness of the absence of something." Topdown attention (at least, in its high-level form) always implies some form of consciousness, even if only consciousness of the absence of the thing one is focusing on or is looking for. Let's consider some phenomena that are usually thought to support the view that there can be top-down attention in the absence of consciousness.

\section{Motion-induced blindness}

Motion-induced blindness (MIB) is a visual illusion in which a perceptually salient stationary visual stimulus repeatedly disappears (and subsequently reappears) when superimposed on a field of moving distracters. Target disappearance in MIB is influenced by attention. Schölvinck and Rees (2009) showed (Experiment 1) that directing spatial attention to an MIB target directly increases its probability of disappearance (compared to an unattended MIB target). Conversely (Experiment 2), increasing the attentional load in a central task unrelated to MIB (for example, performing a conjunction-detection task on the stream of stimuli presented at fixation), decreased the number of disappearances and reappearances of the MIB target.

According to Schölvinck and Rees (2009), these findings appear counterintuitive because, typically, the effect of spatial attention 
is to strengthen the representation of a stimulus, whereas they found that spatial attention directed to an MIB target increased the probability of it disappearing. van Boxtel et al. (2010a) interpret these findings - as well as similar ones - as evidence that the effects of attention and consciousness can potentially be opposed to each other: "the more subjects try to see some stimulus by paying attention to, the less visible it becomes!" (van Boxtel et al., 2010a, p. 7).

In my view, the fact that paying more attention to the MIB target increases the probability of its disappearance from consciousness, simply confirms how vision works: the more you look at something, the more you see. The relevant aspect here is that that "something" is a visual illusion - MIB - and not what one usually sees daily (a familiar sight, a common object, etc.): that is, the content of visual perception is the illusion itself. Contrary to van Boxtel et al.'s (2010a) interpretation, I think that Schölvinck and Rees's (2009) findings show that the more you look at a MIB target, the more visible the illusion becomes! In other words, they can be considered as a case of top-down attention with consciousness of the absence of something ${ }^{3}$.

\section{OVERLOOKING THE EXISTENCE OF THE VARIOUS FORMS OF ATTENTION AND CONSCIOUSNESS: BLINDSIGHT}

A very interesting experiment with a blindsight subject, G.Y., which seems to support the view that there can be top-down attention in the absence of any form consciousness (at least, in the blind area of the subject), was reported by Kentridge et al. (1999). Blindsight subjects are perceptually blind in a certain area of their visual field: they deny having any awareness whatsoever of any visual stimuli presented in that area, or they acknowledge only limited awareness of some phenomena within the blind area, such as movement, but not visual percept. Despite this fact, they are able to discriminate and localize visual stimuli presented in the blind area at levels significantly above chance. In their experiment, Kentridge et al. (1999) adopted a cue-target paradigm. A target, which could appear in one of two possible locations, was presented to the subject; the target was preceded by a cue which might or might not indicate the correct location of the target; the subject was instructed to report - upon hearing an auditory tone that followed the presentation of the visual cue signaling the probable target location - whether or not a visual target had accompanied the presentation of the auditory tone, guessing if necessary, and then to make a second response indicating whether he had had any experience whatsoever. Before the start of each experiment the subject was given instructions indicating the two possible target locations, and in which location the target was more likely to appear. Two main different cueing methods were used in the various experiments in order to investigate whether attention and awareness are inextricably linked or whether there can be endogenous, voluntary attention in the absence of awareness: in the central cueing experiment and in the direct peripheral cueing experiments, the

\footnotetext{
${ }^{3}$ Another example of how it is possible for us to be aware that we are not aware of something, is provided by the phenomenon of the physiological blindspot, that is, the fact that a part of the field of vision cannot be perceived because of the lack of light-detecting photoreceptor cells on the optic disc of the retina where the optic nerve passes through it (see Mole, 2008).
}

target was more likely to appear at the location indicated by the cue than at the other location; in the indirect peripheral cueing experiments, the target was more likely to appear at the location opposite to the one indicated by the cue than at the location indicated by the cue. It is known that indirect cues require voluntary, endogenous attention as opposed to automatic, exogenous attention: in fact, the former imply the application of an arbitrary rule (as opposed to an automatic one) relating the cue and the target location, and the suppression of automatic, exogenous orienting of attention to the cue location. Moreover, it is generally maintained that endogenous, voluntary orienting requires conscious awareness (Posner, 1994). Consequently, Kentridge et al. (1999) predicted that if G.Y. could use the indirect peripheral cue to re-orient his attention, and yet remain unaware of the cue, they would demonstrate that there could be endogenous attention in the absence of consciousness. Indeed, their experiments showed that when an indirect peripheral cue was used, G.Y. could direct voluntary, endogenous attention within his blind field, despite being unaware of the cue he used (upon being questioned after each test on whether he had had any experience whatsoever, he answered that he had had no awareness of any cues). Therefore, Kentridge et al. (1999) concluded that "the spatial selection of information by an attentional mechanism and its entry into conscious experience cannot be one and the same process."

I think that Kentridge et al.'s (1999) findings cannot be straightforwardly interpreted as evidence of top-down attention without consciousness, but rather that they deserve further and more careful examination. In this respect, some alternative interpretations have been proposed.

According to a first alternative interpretation, the fact that G.Y. verbally reported that he had had no awareness of cues does not automatically imply that he had had no conscious experience of anything. As Bartolomeo et al., 2007, p. 157) state: "although an appropriate verbalization can be considered as a reliable indicator of conscious processing (...), the converse is not necessarily true." Indeed, it is possible to distinguish, as the phenomenological tradition has proposed, between "spoken" and "acted" forms of perception, that is, between a high-order, reflective form of consciousness, and a primary, direct form of consciousness (Bartolomeo et al., 2007; Bartolomeo, 2008). Evidence of a dissociation between these two forms of consciousness comes from neuropsychological studies of brain-damaged patients (for example, an amnesic patient with anosognosia who is able to intellectually acknowledge the presence of his deficits, as well as his incapacity to directly appreciate them: for a review, see Bartolomeo and Dalla Barba, 2002; Bartolomeo et al., 2007), and from psychological observations (consider for example the case in which people observing an array of letters for a very short time are aware of having seen letters but can only name some of them). Therefore, from this viewpoint, it is plausible to interpret Kentridge et al.'s (1999) finding as a case of endogenous attention without reflective (autonoetic) consciousness, but with direct, primary (anoetic) consciousness.

According to a second alternative interpretation - which incidentally Kentridge et al.'s, 1999, p. 1810) themselves seem to suggest when they acknowledge that: "it is clear that, while the direction of attention toward a stimulus may be necessary if it 
is to reach awareness (...), attention is not sufficient for awareness," - the fact that G.Y. could re-orient his attention does not automatically mean that he could fully take advantage of all the processes and neural mechanisms entailed and elicited by endogenous attention. According to this view, attention is not so much a unitary process or entity, as a complex control system, or a set of coordinated processes; therefore it is possible to explain G.Y.'s behavior as the result of a partial working of such a complex control system. This is exactly the kind of explanation of Kentridge et al.'s (1999) experiments that has been given by Taylor (2008). According to Taylor, the result of Kentridge et al.'s (1999) experiments can be understood in terms of a corollary discharge and attention feedback amplification of the target stimulus. This would allow a response to be made through an automatic route from the partially activated sensory buffer, so as to be more successful than chance, without any need for the visual buffer to be so strongly activated as to lead to awareness.

Finally, De Brigard and Prinz (2010) explain Kentridge et al.'s (1999) findings by resorting to the distinction between spatial attention, that is, attention to a region of space, and attentional modulation of perceptual representations. In their view, in the case of spatial cueing, a two-stage process occurs: firstly, attention is shifted to the region of space indicated by the visual cue, and secondly, if anything is visible in that space, that thing gets visually represented and modulated by attention. Only the latter stage would involve consciousness, not the former. Consequently, a shift of attention to a region of unoccupied space would not result in any conscious visual percept. In Kentridge et al.'s (1999) study, only the first stage could occur, causing G.Y. to attend to a region of space, but the second stage could not occur because of G.Y.'s lesion in his primary visual cortex. De Brigard and Prinz (2010) also provide three possible reasons why the spatial cue could facilitate G.Y.'s performance: (1) attending to a region of space may lower signaldetection thresholds for stimuli presented subsequently in that region; (2) the spatial attention may cause receptive fields in the region to expand, with the subsequent increase of neural resources for the ensuing presented target; (3) spatial attention may prime the blindsighter for behavior responses in the attended region.

It should be noted, however, that De Brigard and Prinz's (2010) explanation could be questioned on the grounds that it seems to be more suitable for a case involving the automatic, exogenous orienting of attention (by a direct peripheral cue), than for a case involving the voluntary, endogenous orienting of attention (by an indirect peripheral cue).

\section{CAN ANY KIND OF ATTENTION BE DISSOCIATED FROM CONSCIOUSNESS? ONLY LOW-LEVEL ATTENTION (PRELIMINARY ATTENTION), BUT NOT HIGH-LEVEL TOP-DOWN ATTENTION}

According to Velmans (1991, p. 665), focal-attentive processing provides the necessary condition for conscious awareness, and there cannot be consciousness without attention: consciousness results from focal-attentive processing as a form of output. However, he affirms that, "in principle, it might be possible to obtain evidence of focal-attentive processing in the absence of awareness of what is being processed": that is, attention and consciousness are not the same thing, and in principle can be dissociated, because there can be attentional processing without consciousness, even though he recognizes that: "in practice, however, a complete dissociation of consciousness from focal-attentive processing is difficult to achieve."

Velmans (1991) aim is to confute the conventional assumption by psychologists that "preconscious" processing is identical to "pre-attentive" processing and "conscious" processing is identical to "focal-attentive" processing. This assumption implies that "preconscious/pre-attentive" processing is fast, automatic, involuntary, and restricted to simple, familiar stimuli, whereas "conscious/focal-attentive" processing is slow, flexible, voluntary, and subject to intentional control. Velmans' confutation is based on evidence that preconscious processing is not inflexible and limited to simple, well-learned stimuli: he provides many examples of preconscious analysis of novel and complex phrases and sentences, implicit learning, preconscious selection and choice, unconscious control of complex, novel motor adjustments, and unconscious planning. Consequently, it would be misleading for him to think of the preconscious-unconscious processing of stimuli as non-attended or pre-attentive: preconsciously processed stimuli, being subject to sophisticated, elaborated analysis, receive attentional resources, although they may not enter consciousness. Moreover, there is evidence (Kahneman and Chajczyk, 1983) that "involuntary, preconscious" analysis of stimuli is not necessarily effortless, and that it draws on, and competes for, limited processing resources, which confirms the involvement of attentional resources in preconscious processing (see also Lavie, 1995). Therefore, rather than speaking of non-attended or pre-attentive processing (vs. focal-attentive processing), it would be better to speak of preliminary attention (vs. focal attention; Velmans, 1991. p. 655).

I think that most of the studies which Velmans cites, aimed at showing a possible dissociation between focal attention and consciousness, do not show that there can be focal-attentive processing without consciousness, but rather that preliminary attention and consciousness can be dissociated. In fact, whether they refer to dichotic listening tasks and shadowing tasks (Treisman, 1964a,b; Lackner and Garrett, 1973; MacKay, 1973), visual masking experiments (Marcel, 1980, 1983), Stroop effect, implicit learning (Hartman et al., 1987; Nissen and Bullemer, 1987), or control of action, they all only show that stimuli can be preconsciously processed if they are given at least a minimal level of attention.

Indeed, as observed by some authors (Neuman, 1984; Holender, 1986; Logan, 1995), in these cases, as well as in others such as the flanker compatibility effect or negative priming effect (Tipper, 1985), subjects do pay a certain, even if marginal, level of attention to the to-be-ignored, unwanted stimuli, even though they are instructed not to pay attention to them, or are prevented from paying attention to them. This marginal level of attention can be brought about and maintained in various ways: one of the most common is by widely distributing the focus of attention. For example, McCormick (1997, p. 178), commenting on his finding that an exogenous cue presented below a subjective threshold of awareness captures attention automatically and without awareness, explicitly observes that "this finding and the issue of the automaticity of exogenous orienting is limited to specific experimental conditions (...). In my experiments, the observers' attention was likely distributed widely over the visual field in anticipation of the pending 
cue and target events, thus it could be involuntarily attracted to the cue."

For example, the fact that amnesic patients and normal subjects, when exposed to successive exemplars of recurring patterns of which they were unaware, can implicitly learn those patterns without spontaneously noting any repeated sequence (Hartman et al., 1987; Nissen and Bullemer, 1987), does not imply that they have used their focal attention to learn those patterns: in fact, they have been instructed to pay attention to the single items composing each pattern, and not to the recurring pattern. Therefore, it is inappropriate to affirm that there is focal-attentive processing of a pattern in the absence of awareness of that pattern, because what subjects attentively process is not so much the pattern as the single items of the pattern. Most probably instead, they were able to learn the patterns because they spent a marginal amount of their attentional resources on them: such a marginal amount that they could not consciously realize what they were doing, even though it was sufficient to make them learn the patterns.

An author who does not fail to notice that only preliminary or low-level (but not high-level focal) attention can be dissociated from consciousness is Damasio (1999). In his definition, consciousness is the "umbrella term for the mental phenomena that permit the strange confection of you as observer or knower of the things observed, of you as owner of thoughts formed in your perspective, of you as potential agent on the scene" (Damasio, 1999, p. 127). Throughout his work, consciousness is seen as the main reason for the feeling we have of ourselves as the subject of our own actions, that is, for the fact that we sense that what we are doing is done by us, and not by someone else.

In his opinion, some diseases, such as akinetic mutism, epileptic automatism and advanced stages of Alzheimer's diseases, demonstrate that there can be fleeting, low-level attention without consciousness. Evidence of the dissociation between low-level attention and consciousness is given by patients who, while exhibiting some elementary signs of attention such as the ability to form sensory images of objects and execute accurate movements relative to those images, do not develop any sense of self, of an individual organism wishing, considering, wanting, of a person with a past and a future. Moreover, they do not show any sign of emotion either. Finally, Damasio (1999, p. 91) points out that only a kind of attention that is high-level, extended in time and focused on appropriate objects is indicative of consciousness. Despite not specifying exactly what the difference is between low-level and high-level attention, these findings seem to support nonetheless the hypothesis that high-level focal attention cannot be dissociated from consciousness.

Summarizing the data provided by Velmans (1991) and Damasio's (1999) works, we can say that consciousness can be dissociated only from low-level attention (or, as Velmans calls it, preliminary attention), whether of an endogenous or exogenous kind: high-level top-down attention cannot be dissociated from consciousness. The determination of the threshold level at which attention can be dissociated from consciousness is obviously a matter of empirical investigation. However, in principle, it cannot be excluded that attention, when applied at nearthreshold, or when not fully deployed because the stimulus duration does not allow for it, gives rise to unpredictable, paradoxical phenomena (as it frequently happens in the psychological field: see for example the phenomena of temporal displacement and continuous displacement described in Vicario, 2005) ${ }^{4}$.

\section{Afterimage}

Experiments in afterimage studies are sometimes used as evidence that attention and consciousness can be fully dissociated because they produce opposite effects. For example, van Boxtel et al.'s, 2010b, p. 8886) report some experiments on the formation of afterimage perception as evidence that "selective attention and stimulus consciousness have separable effects on perception (...) and, in the context of afterimages, may even have opposite effects." In van Boxtel et al. (2010b), attention and awareness were independently manipulated during the afterimage induction phase, while the effects of these manipulations were measured in the afterimage perception phase. Attention to the afterimage inducer was manipulated by employing an attention-distracting task at fixation (i.e., the central task). This task could be easy or hard, ensuring identical visual input while manipulating the levels of attention available to the afterimage inducer. The conscious visibility of the inducer stimulus was manipulated independently of attention by means of CFS, a form of inter-ocular suppression (i.e., presenting a very salient object in one eye that completely suppresses the afterimage inducer in the other eye). With the suppression present, the Gabor patch inducing the afterimage was not perceived. This $2 \times 2$ design allowed for a full-factorial comparison (i.e., high attention/visible, low attention/visible, high attention/invisible, and low attention/invisible). According to van Boxtel et al. (2010b), these data show that paying more attention to the inducer invariably shortens afterimage duration, while increasing the visibility (i.e., consciousness) of the inducer increases afterimage duration vs. invisibility.

The interpretation of van Boxtel et al.'s (2010b) experiments as evidence that attention and consciousness can be fully dissociated is questionable for a couple of reasons at least.

Firstly, CFS is a technique that, physically perturbing the transmission of the information along the way from the stimulus to the brain, alters the normal processing of visual stimuli. As such, it cannot be excluded either that top-down attention is not given sufficient time to be fully deployed and to finalize its course, or that, even if top-down attention is fully deployed, only part of the stimulus is processed. I am not denying that the stimulus is not processed: empirical evidence clearly shows that the invisible stimulus is somehow processed in human primary visual cortex (Bahrami et al., 2007; Watanabe et al., 2011). What I claim is that the process initiated by top-down attention is partly interrupted or modified, and that this does not allow for the stimulus to be consciously perceived. Therefore, with respect to the stimulus, the situation can be assimilated to a situation in which there is only marginal, low-level attention, although one has tried to deploy high-level top-down attention. As such, the data in the invisible condition (when the inducer is suppressed by CFS) cannot be used to support the view that paying more attention to the inducer decreases the duration of its afterimage.

\footnotetext{
${ }^{4}$ For a commentary of Vicario’s book, see Marchetti (2006).
} 
Secondly, it could be claimed that the afterimage is the product not so much of attending as of consciously perceiving what is attended. In this view, van Boxtel et al.'s (2010b) findings would not show that "attention produces weaker afterimages"; rather they would show that "attention via consciousness produces weaker afterimages." Indeed, it is conceivable that selectively attending to a stimulus produces conscious perception of the stimulus, which, in turn, can produce some other (conscious or unconscious) effect (for example, a decision to search for another stimulus, or to continue to observe the previous stimulus). In the afterimage case, this would entail that attending to a Gabor patch first produces a conscious perception of the Gabor patch, which in turn produces some effect (namely, an afterimage having certain properties, such as being weaker and shorter than an afterimage produced by an unattended Gabor patch). Here, the afterimage would be a direct consequence not so much of selectively attending, as of consciously perceiving the Gabor patch. Without such conscious perceptions, afterimages might not possess any of the specific properties (namely, the characteristics of being weak and short) they acquire thanks to conscious processing. Consequently, also van Boxtel et al.'s statement that selective attention produces weaker afterimages should be reformulated: selective attention (possibly) produces weaker afterimages indirectly and via conscious perception of the attended stimulus.

This interpretation does in fact conflict with what the experiments seem to show, i.e., that increasing the visibility (i.e., consciousness) of the inducer increases afterimage duration vs. invisibility. However, it should be noted that the experiments say nothing about the level of visibility in the visible trials when different levels of attention are paid. That is, they do not measure whether stimuli are equally or differently visible in the two different attentional conditions (low vs. high attention). It might turn out, for example, that when the inducer stimulus is highly attended, it is more visible than when the stimulus is not/slightly attended. After all, strength of afterimages has been shown (Baijal and Srinivasan, 2009) to be modulated by the spatial spread of attention and the specific attentional strategy (for example, distributed vs. focal attention) that is adopted to perform the task and yields different types of awareness (for example, background consciousness vs. object consciousness). As Baijal and Srinivasan's (2009) experiments show, afterimage duration is significantly longer when the identification and counting task is performed with small compared to large letters, local compared to global stimuli, small compared to local stimuli, and global compared to large stimuli. Therefore, until it is determined how the level of visibility in the visible trials changes with the various levels of attention, it cannot be completely ruled out that increasing the visibility (via the increase of the amount of applied attention) produces weaker afterimages.

\section{ATTENTION AND CONSCIOUSNESS ARE NOT THE SAME THING}

The fact that attention is necessary for consciousness and that high-level top-down attention always implies some form of consciousness does not imply that attention is the same thing as consciousness.
Firstly, not always does attention generate conscious phenomena. As we have seen, attention can also generate and modulate unconscious ones (Naccache et al., 2002; Montaser-Kousari and Rajimehr, 2005; Sumner et al., 2006; Bahrami et al., 2008).

Secondly, as some authors have suggested, consciousness also needs some other components. For example, according to Srinivasan (2008), components such as expectations and capacity of anticipation are necessary for consciousness: indeed, what IB and $\mathrm{CB}$ experiments seem to show is that when the observers fail to detect changes this may be due to their expectations of a stable world and inability to anticipate the stimulus (in the sense that, for example, observers do not expect people to suddenly change into someone else). My model of consciousness includes additional components such as sense-organs, somatosensory organs, working memory, and a schema of self (Marchetti, 2010). It should be noted, however, that it is always possible to incorporate such components directly into the attentional system: Knudsen's (2007) proposes that attention reflects the combined contributions of four distinct processes: working memory, competitive selection, top-down sensitivity control, and automatic filtering for salient stimuli.

Thirdly, attention and consciousness can also be distinguished from a functional point of view. Consciousness can be described as resulting from the activity performed by attention (see also Velmans, 1991), and more precisely from the application of attention to the other organs or to attention itself, and the consequent modulation of the state of the organ of attention (Marchetti, 2010). This difference is partly captured by Baars' (1997, p. 364) description of attention as something more active than consciousness, and of consciousness as the result of this activity: "It is as if attention resembles selecting a desired television program, and consciousness is what appears on screen." The functional distinction between attention and consciousness has also been highlighted, but in different terms, by van Boxtel et al. (2010a), who conceive of selective, focal attention as an analyzer and consciousness as a synthesizer.

Therefore, my answer to the question of whether there can be human consciousness at all, as we know it, without sense-organs, somatosensory organs, working memory, and all the connections linking one component to the others and to attention, is obviously no: attention alone is not sufficient and the other components are also necessary.

\section{CONCLUSION}

In this paper, we have seen that the idea that there can be consciousness without some form of attention, and high-level top-down attention without consciousness, originates from a failure to notice the varieties of forms that top-down attention and consciousness can assume. Overlooking the fact that both attention and consciousness can assume a variety of forms may lead one to: mistake the effects of a form of attention for the effects of another form of attention; ignore that subjects can adopt different attentional strategies depending on the specific task they are required to perform; fail to notice that different attentional strategies may yield different types of awareness.

Once the varieties of forms of attention and consciousness are taken into consideration, the necessity of attention for 
consciousness can hardly be denied. As we have seen, there can be low-level attention or preliminary attention without consciousness, but there cannot be high-level top-down attention without consciousness. High-level top-down attention always implies some form of consciousness. On the contrary, low-level or preliminary attention can either imply consciousness (such as when the cocktail party effect occurs) or absence of consciousness. Even if

\section{REFERENCES}

Alvarez, G. A. (2011). Representing multiple objects as an ensemble enhances visual cognition. Trends Cogn. Sci. (Regul. Ed.) 15, 122-131.

Baars, B. J. (1997). Some essential differences between consciousness and attention, perception, and working memory. Conscious. Cogn. 6 , 363-371.

Bahrami, B., Carmel, D., Walsh, V., Rees, G., and Lavie, N. (2008). Unconscious orientation processing depends on perceptual load. J. Vis. $8,12,1-10$.

Bahrami, B., Lavie, N., and Rees, G. (2007). Attentional load modulates responses of human primary visual cortex to invisible stimuli. Curr. Biol. 17, 509-513.

Baijal, S., and Srinivasan, N. (2009). Types of attention matter for awareness: a study with color afterimages. Conscious. Cogn. 18, 1039-1048.

Bartolomeo, P. (2008). Varieties of attention and of consciousness: evidence from neuropsychology. Psyche 14. Available at: http://theassc.org/vol_14_2008

Bartolomeo, P., and Dalla Barba, G. (2002). Varieties of consciousness. Behav. Brain Sci. 25, 331-332.

Bartolomeo, P., Decaix, C., and Siéroff, E. (2007). The phenomenology of endogenous orienting. Conscious. Cogn. 16, 144-161.

Becker, M. W., Pashler, H., and Anstis, S. M. (2000). The role of iconic memory in change-detection tasks. Perception 29, 273-286.

Block, N. (1996). How can we find the neural correlate of consciousness. Trends Neurosci. 19, 456-459.

Brown, S. W. (1985). Time perception and attention: the effects of prospective versus retrospective paradigms and task demands on perceived duration. Percept. Psychophys. $38,115-124$.

Carrasco, M. (2011). Visual attention. Vision Res. 51, 1484-1525.

Carrasco, M., Fuller, S., and Ling, S. (2008). Transient attention does increase perceived contrast of suprathreshold stimuli: a reply to
Prinzmetal, Long, and Leonhardt (2008). Percept. Psychophys. 70, 1151-1164.

Carrasco, M., Ling, S., and Read, S. (2004). Attention alters appearance. Nat. Neurosci. 7, 308-313.

Cartwright-Finch, U., and Lavie, N. (2007). The role of perceptual load in inattentional blindness. Cognition $102,321-340$

Chica, A. B., Lasaponara, S., Chanes, L., Valero-Cabré, A., Doricchi, F., Lupiáñez, J., and Bartolomeo, P. (2011). Spatial attention and conscious perception: the role of endogenous and exogenous orienting. Atten. Percept. Psychophys. 73, 1065-1081.

Coull, J. T., Vidal, F., Nazarian, B., and Macar, F. (2004). Functional anatomy of the attentional modulation of time estimation. Science 303, 1506-1508.

Damasio, A. (1999). The Feeling of What Happens. Body and Emotions in the Making of Consciousness. New York: Harcourt Brace \& Co.

De Brigard, F., and Prinz, J. (2010). Attention and consciousness. Wiley Interdiscip. Rev. Cogn. Sci. 1, 51-59.

Dehaene, S., Changeux, J. P., Naccache, L., Sackur, J., and Sergent, C. (2006). Conscious, preconscious, and subliminal processing: a testable taxonomy. Trends Cogn. Sci. (Regul. Ed.) 10, 204-211.

Demeyere, N., and Humphreys, G. (2007). Distributed and focused attention: neuropsychological evidence for separate attentional mechanisms when counting and estimating. J. Exp. Psychol. Hum. Percept. Perform. 33, 1076-1088.

Di Lollo, V., Kawahara, J.-I., Ghorashi, attentional blink: resource depletion or temporary loss of control? Psychol. Res. 69, 191-200.

Edelman, G. M. (1989). The Remembered Present: A Biological Theory of Consciousness. New York: Basic Books.

Hardcastle, V. G. (1997). Attention versus consciousness: a distinction with a difference. Cogn. Stud. Bull. Jpn. Cogn. Sci. Soc. 4, 56-66. S. M. S., and Enns, J. T. (2005). The

attention cannot be considered the same thing as consciousness, some form of attention is always necessary for consciousness.

\section{ACKNOWLEDGMENTS}

I am grateful to the two reviewers and the editor for their insightful comments, which helped me better frame my thought, and to Wendy Piemonte for the English revision of the article.

Hartman, M., Knopman, D. S., and Nissen, M. J. (1987). Implicit learning of new verbal associations. J. Exp. Psychol. Learn. Mem. Cogn. 15 1070-1082.

Hicks, R. E., Miller, G. W., Gaes, G., and Bierman, K. (1977). Concurrent processing demands and the experience of time-in-passing. Am. J. Psychol. 89, 719-730.

Hicks, R. E., Miller, G. W., Kinsbourne, M. (1976). Prospective and retrospective judgments of time as a function of amount of information processed. Am. J. Psychol. 90 431-446.

Holender, D. (1986). Semantic activation without consciousness in dichotic listening, parafoveal vision and visual masking. A survey and appraisal. Behav. Brain Sci. 9, 1-66.

Hsieh, P.-J., Colas, J. T., and Kanwisher, N. (2011). Pop-out without awareness: unseen feature singletons capture attention only when top-down attention is available. Psychol. Sci. 22 1220-1226.

Iwasaki, S. (1993). Spatial attention and two modes of visual consciousness. Cognition 49, 211-233.

James, W. (1890). The Principles of Psychology. New York: Holt.

Kahneman, D. (1973). Attention and Effort. Englewood Cliffs, NJ: Prentice Hall.

Kahneman, D., and Chajczyk, D. (1983). Test of the automaticity of reading: diluition of Stroop effects by colorirrelevant stimuli. J. Exp. Psychol. Hum. Percept. Perform. 9, 497-509.

Kentridge, R. W., Heywood, C. A. and Weiskrantz, L. (1999). Attention without awareness in blindsight. Proc. R. Soc. Lond. B Biol. Sci. 266, 1805-1811.

Knudsen, E. I. (2007). Fundamental components of attention. Annu. Rev. Neurosci. 30, 57-78.

Koch, C., and Tsuchiya, N. (2006). Attention and consciousness: two distinct brain processes. Trends Cogn. Sci. (Regul. Ed.) 11, 16-22.

Koivisto, M., Kainulainen, P., and Revonsuo, A. (2009). The relationship between awareness and attention: evidence from ERP responses. Neuropsychologia 47, 2891-2899.
Koivisto, M., and Revonsuo, A. (2010). Event-related brain potential correlates of visual awareness. Neurosci. Biobehav. Rev. 34, 922-934.

Kouider, S., de Gardelle, V., Sackur, J., and Dupoux, E. (2010). How rich is consciousness? The partial awareness hypothesis. Trends Cogn. Sci. (Regul. Ed.) 14, 301-307.

La Berge, D. (1995). Attentional Processing. The Brain's Art of Mindfulness. Cambridge: Harvard University Press.

Lackner, J., and Garrett, M. F. (1973). Resolving ambiguity: effect of biasing context in the unattended ear. Cognition 1, 359-372.

Lamme, V. A. F. (2003). Why visual attention and awareness are different. Trends Cogn. Sci. (Regul. Ed.) 7, 12-18.

Landman, R., Spekreijse, H., and Lamme, V. A. F. (2003). Large capacity storage of integrated objects before change blindness. Vision Res. 43, 149-164.

Lavie, N. (1995). Perceptual load as a necessary condition for selective attention. J. Exp. Psychol. Hum. Percept. Perform. 21, 451-468.

Lavie, N. (2006a). "Attention and consciousness," in The Blackwell Companion to Consciousness, eds M. Velmans and S. Schneider (Oxford: Blackwell Publishing Ltd.), 489-503.

Lavie, N. (2006b). The role of perceptual load in visual awareness. Brain Res. 1080, 91-100.

Li, F. F., VanRullen, R., Koch, C., and Perona, P. (2002). Rapid natural scene categorization in the near absence of attention. Proc. Natl. Acad. Sci. U.S.A. 99, 9596-9601.

Liu, T., Abrams, J., and Carrasco, M. (2009). Voluntary attention enhances contrast appearance. Psychol. Sci. 20, 354-362.

Logan, G. D. (1995). Linguistic and conceptual control of visual spatial attention. Cogn. Psychol. 28, 103-174.

Mack, A., and Rock, I. (1998). Inattentional Blindness. Cambridge: MIT Press. 
MacKay, D. G. (1973). Aspects of the theory of comprehension, memory and attention. Q. J. Exp. Psychol. 25, 22-40.

Marcel, A. J. (1980). "Conscious and preconscious recognition of polysemous words: locating the selective effects of prior verbal context," in Attention and Performance VII, ed. R. S. Nickerson (Hillside, NJ: Lawrence Erlbaum Associates), 435-457.

Marcel, A. J. (1983). Conscious and unconscious perception: experiments on visual masking and word recognition. Cogn. Psychol. 15, 197-237.

Marchetti, G. (2006). Commentary on Giovanni Bruno Vicario's Il tempo. Saggio di psicologia sperimentale. Available at: http://www.mind-consciousnesslanguage.com/comm.htm

Marchetti, G. (2010). Consciousness, Attention and Meaning. New York: Nova Science Publishers.

Mattes, S., and Ulrich, R. (1998). Directed attention prolongs the perceived duration of a brief stimulus. Percept. Psychophys. 60, 1305-1317.

McCormick, P. A. (1997). Orienting attention without awareness. J. Exp. Psychol. Hum. Percept. Perform. 23, 168-180.

McMains, S. A., and Somers, D. C. (2004). Multiple spotlights of attentional selection in human visual cortex. Neuron 42, 677-686.

Merikle, P. M., Smilek, D., and Eastwood, J. D. (2001). Perception without awareness: perspectives from cognitive psychology. Cognition 79, 115-134.

Mole, C. (2008). Attention and consciousness. J. Conscious. Stud. 15, 86-104.

Montaser-Kousari, L., and Rajimehr, R. (2005). Subliminal attentional modulation in crowding condition. Vision Res. 45, 839-844.

Moray, N. (1959). Attention and dichotic listening: affective cues and the influence of instructions. Q. J. Exp. Psychol. 11, 56-60.

Most, S. B., Scholl, B. J., Clifford, E. R., and Simons, D. J. (2005). What you see is what you set: sustained inattentional blindness and the capture of awareness. Psychol. Rev. 112, 217-242.

Naccache, L., Blandin, E., and Dehaene, S. (2002). Unconscious masked priming depends on temporal attention. Psychol. Sci. 13, 416-424.

Nakayama, K., and Mackeben, M. (1989). Sustained and transient components of focal visual attention. Vision Res. 29, 1631-1647.

Neuman, O. (1984). "Automatic processing. A review of recent findings and a plea for an old theory," in Cognition and Motor Processes, eds W. Printz and A. Sanders (Berlin: Springer), 255-293.

Nissen, M. J., and Bullemer, P. (1987). Attentional requirements of learning: evidence from performance measures. Cogn. Psychol. 19, 1-32.

Olivers, C. N., and Nieuwenhuis, S. (2005). The beneficial effect of concurrent task-irrelevant mental activity on temporal attention. Psychol. Sci. 16, 265-269.

O’Regan, J. K., and Noë, A. (2001). A sensorimotor account of vision and visual consciousness. Behav. Brain Sci. 24, 939-1031.

Pashler, H. E. (1998). The Psychology of Attention. Cambridge: MIT.

Posner, M. I. (1994). Attention: the mechanisms of consciousness. Proc. Natl. Acad. Sci. U.S.A. 91, 7398-7403.

Posner, M. I. (2008). Attentional networks and the semantics of consciousness. Psyche 14. Available at: http://theassc.org/vol_14_2008

Prinzmetal, W., Long, V., and Leonhardt, J. (2008). Involuntary attention and brightness contrast. Percept. Psychophys. 70, 1139-1150.

Reddy, L., Wilken, P., and Koch, C. (2004). Face-gender discrimination is possible in the near-absence of attention. J. Vis. 4, 106-117.

Rensink, R. A., O’Regan, J. K., and Clark, J. J. (1997). To see or not to see: the need for attention to perceive changes in scenes. Psychol. Sci. 8, 368-373.

Ro, T., Russel, C., and Lavie, N. (2001). Changing faces: a detection advantage in the flicker paradigm. Psychol. Sci. 12, 94-99.

Schneider, K. A. (2006). Does attention alter appearance? Percept. Psychophys. 68, 800-814.

Schneider, K. A., and Komlos, M. (2008). Attention biases decisions but does not alter appearance. J. Vis. 8, 1-10.

Schölvinck, M. L., and Rees, G. (2009). Attentional influences on the dynamics of motion-induced blindness. J. Vis. 9, 1-9.
Shapiro, K. L., Arnell, K. M., and Raymond, J. E. (1997). The attentional blink. Trends Cogn. Sci. (Regul. Ed.) 1, 291-296.

Shapiro, K. L., Raymond, J. E., and Arnell, K. M. (1994). Attention to visual pattern information produces the Attentional blink in rapid serial visual presentation. J. Exp. Psychol. Hum. Percept. Perform. 20, 357-371.

Shore, D. I., Spence, C., and Klein, R. M. (2001), Visual prior entry. Psychol. Sci. 12, 205-212.

Simons, D. J., and Levin, D. T. (1997) Change blindness. Trends Cogn. Sci. 1, 261-267.

Simons, D. J., and Levin, D. T. (1998) Failure to detect changes to people during a real-world interaction. Psychon. B. Rev. 5, 644-649.

Srinivasan, N. (2008). Interdependence of attention and consciousness. Prog. Brain Res. 168, 65-75.

Srinivasan, N., Srivastava, P., Lohani, M., and Baijal, S. (2009). Focused and distributed attention. Prog. Brain Res. 176, 87-100.

Sumner, P., Tsai, P.-C., Yu, K., and Nachev, P. (2006). Attentional modulation of sensorimotor processes in the absence of perceptual awareness. Proc. Natl. Acad. Sci. U.S.A. 103 10520-10525.

Taylor, J. G. (2008). On the relation between attention and consciousness. Psyche 14. Available at: http://theassc.org/vol_14_2008

Taylor, J. G., and Fragopanagos, N. (2007). Resolving some confusions over attention and consciousness. Neural Net. 20, 993-1003.

Tipper, S. P. (1985). The negative priming effect: inhibitory effects of ignored primes. Q. J. Exp. Psychol. 37A, 571-590.

Treisman, A. (1964a). Verbal cues, language and meaning in attention. Am. J. Psychol. 77, 206-214.

Treisman, A. (1964b). The effect of irrelevant material on the efficiency of selective listening. Am. J. Psychol. 7, 533-546.

Treisman, A. (2006). How the deployment of attention determines what we see. Vis. Cogn. 14, 411-443.

Treisman, A., and Gelade, G. (1980). A feature-integration theory of attention. Cogn. Psychol. 12, 97-136.

Tulving, E. (1985). Memory and consciousness. Can. Psychol. 26, 1-12.

Umiltà, C. (1994). "Attenzione e coscienza," in Manuale di psicologia generale, ed. P. Legrenzi (Bologna: Il Mulino), 185-250.

van Boxtel, J. J. A., Tsuchiya, N., and Kock, C. (2010a). Consciousness and attention: on sufficiency and necessity. Front. Psychol. 1:1-13. doi:10.3389/fpsyg.2010.00217

van Boxtel, J. J. A., Tsuchiya, N., and Kock, C. (2010b). Opposing effects of attention and consciousness on afterimages. Proc. Natl. Acad. Sci. U.S.A. 107, 8883-8888.

Vandekerckhove, M., and Panksepp, J. (2009). The flow of anoetic to noetic and autonoetic consciousness: a vision of unknowing (anoetic) and knowing (noetic) consciousness in the remembrance of things past and imagined futures. Conscious. Cogn. 18, 1018-1028.

Velmans, M. (1991). Is human information processing conscious? Behav. Brain Sci. 14, 651-726.

Vicario, G. B. (2005). Il tempo. Saggio di psicologia sperimentale. Bologna: Il Mulino.

Watanabe, M., Cheng, K., Murayama, Y., Ueno, K., Asamizuya, T., Tanaka, K. and Logothetis, N. (2011). Attention but not awareness modulates the BOLD signal in the human V1 during binocular suppression. Science 334, 829-831.

Wittmann, M. (2011). Moments in time. Front. Integr. Neurosci. 5:66. doi:10.3389/fnint.2011.00066.

Conflict of Interest Statement: The author declares that the research was conducted in the absence of any commercial or financial relationships that could be construed as a potential conflict of interest.

Received: 09 July 2011; accepted: 31 January 2012; published online: 15 February 2012.

Citation: Marchetti G (2012) Against the view that consciousness and attention are fully dissociable. Front. Psychology 3:36. doi: 10.3389/fpsyg.2012.00036

This article was submitted to Frontiers in Consciousness Research, a specialty of Frontiers in Psychology.

Copyright (c) 2012 Marchetti. This is an open-access article distributed under the terms of the Creative Commons Attribution Non Commercial License, which permits non-commercial use, distribution, and reproduction in other forums, provided the original authors and source are credited. 